\title{
Eficiencia de un reactor SBR para la remoción de la materia orgánica presente en el agua residual de una industria de teñido de flores
}

\author{
Alfoncina Restrepo; Diana C. Rodríguez*; Gustavo A. Peñuela \\ Grupo Diagnóstico y Control de la Contaminación (GDCON), Escuela Ambiental, Facultad de Ingeniería, Sede de \\ Investigaciones Universitarias (SIU), Universidad de Antioquia (UdeA), Calle 70 No. 52-21, Medellín, Colombia. \\ *diana.rodriguez@udea.edu.co
}

\begin{abstract}
Resumen
Se realizó la implementación de un reactor SBR (Sequencing Batch Reactor- por sus siglas en inglés y Reactor Secuencial en Discontinuo - en español) para la remoción de la materia orgánica presente en el agua residual de una empresa de teñido de flores. Se analizaron parámetros fisicoquímicos para evaluar la eficiencia del proceso y seguimiento de la biomasa mediante pruebas de sedimentabilidad y observaciones al microscopio. El sistema SBR fue operado usando una fase de llenado estática durante $2 \mathrm{~h}$, una etapa de reacción durante $8 \mathrm{~h}$ ( 4 h de aireación y $4 \mathrm{~h}$ de mezcla) y finalmente una etapa de sedimentación durante $2 \mathrm{~h}$, para un total de 12 horas por ciclo y 2 ciclos al día, y se evaluaron dos colorantes azo (C1 y C2) ampliamente usados en la industria del teñido de flores, variando temporalmente la concentración de la materia orgánica en términos de DQO con valores de 3,0 g / L.d y 7,0 g /L.d. Los resultados experimentales y el análisis estadístico permitió analizar el comportamiento del reactor SBR, concluyendo que ambos colorantes se comportaron estadísticamente iguales en la remoción del carbono orgánico total (COT), con un valor promedio de remoción 92,02\% para C1 y 94,60\% para C2 con una carga de 3,0 g DQO/L.d y 96,69\% para C1 y 98,30\% para C2 con una carga de 7,0 g DQO/L.d. La biomasa presentó un IVL bajo, indicando una buena sedimentabilidad tanto para los ensayos llevados a cabo con $\mathrm{C} 1$ como C2. Finalmente, los microorganismos identificados en la biomasa permitieron corroborar la eficiencia del sistema de tratamiento, dado que abundaron los rotíferos y ciliados fijos con C1 y ciliados libres con C2, los cuales son indicadores de una buena eficiencia en los procesos de tratamiento y a su vez, indicadores de edades de lodos elevadas, lo que contrasta con la edad de lodos empleada en este estudio $(\theta c=15 d)$.
\end{abstract}

Palabras clave: Colorantes; Materia orgánica; Procesos biológicos; Reactor aerobio; Reactor anaerobio. 


\title{
Efficiency of an SBR reactor for the removal of the organic matter present in the wastewater of a flower dyeing industry
}

\begin{abstract}
The implementation of an SBR reactor (Sequencing Batch Reactor- for its acronym in English and Sequential Reactor in Discontinuous- in Spanish) was carried out for the removal of organic matter present in the wastewater of a flower dyeing company. Physicochemical parameters were analyzed to evaluate the efficiency of the process and biomass monitoring through sedimentation tests and microscopic observations. The SBR system was operated using a static filling phase for $2 \mathrm{~h}$, a reaction step for $8 \mathrm{~h}(4 \mathrm{~h}$ of aeration and $4 \mathrm{~h}$ of mixing) and finally a sedimentation step for $2 \mathrm{~h}$, for a total of 12 hours per cycle and 2 cycles per day and two azo dyes ( $\mathrm{C} 1$ and $\mathrm{C} 2$ ) widely used in the flower dyeing industry were evaluated, temporarily varying the concentration of organic matter in terms of COD with values of $3.0 \mathrm{~g} / \mathrm{L} . \mathrm{d}$ and $7.0 \mathrm{~g} / \mathrm{L}$.d The experimental results and statistical analysis allowed to analyze the behavior of the SBR reactor, concluding that both colorants behaved statistically the same in the removal of total organic carbon (TOC), with an average removal value of $92.02 \%$ for $\mathrm{C} 1$ and $94.60 \%$ for $\mathrm{C} 2$ with a loading of $3.0 \mathrm{~g} \mathrm{COD/L.d}$ and $96.69 \%$ for $\mathrm{C} 1$ and $98.30 \%$ for $\mathrm{C} 2$ with a loading of $7.0 \mathrm{~g} \mathrm{COD/L.d} \mathrm{The} \mathrm{biomass} \mathrm{presented} \mathrm{a} \mathrm{low} \mathrm{SVI,} \mathrm{indicating} \mathrm{a} \mathrm{good}$ sedimentability for both the tests carried out with $\mathrm{C} 1$ and $\mathrm{C} 2$. Finally, the microorganisms identified in the biomass allowed to corroborate the efficiency of the treatment system, since rotifers and fixed ciliates with $\mathrm{C} 1$ and free ciliates with C2 abounded, which are indicators of good efficiency in the treatment processes and, in turn, high sludge age indicators, which contrasts with the sludge age used in this study $(\theta c=15 d)$.
\end{abstract}

Keywords: Dyes; Organic material; Biological processes; Aerobic reactor; Anaerobic reactor.

\section{Eficiência de um reator SBR para a remoção da matéria orgânica presente na água residual de uma indústria de tinturas de flores}

\begin{abstract}
Resumo
Foi realizada a implantação de um reator SBR (Sequencing Batch Reactor- por sua sigla em inglês e Sequential Reactor em Discontinuous- em espanhol) para a remoção de matéria orgânica presente no efluente de uma empresa de tinturaria de flores. Parâmetros físico-químicos foram analisados para avaliação da eficiência do processo e monitoramento da biomassa por meio de testes de sedimentação e observações microscópicas. O sistema SBR foi operado usando uma fase de enchimento estático por $2 \mathrm{~h}$, uma etapa de reação por $8 \mathrm{~h}$ ( $4 \mathrm{~h}$ de aeração e $4 \mathrm{~h}$ de mistura) e finalmente uma etapa de sedimentação por $2 \mathrm{~h}$, para um total de 12 horas por ciclo e 2 Foram avaliados ciclos diários e dois corantes azo (C1 e C2) amplamente utilizados na indústria de tingimento de flores, variando temporariamente a concentração de matéria orgânica em termos de DQO com valores de 3,0 g/L.d e 7,0 g/L.d Os resultados experimentais e estatísticos a análise permitiu analisar o comportamento do reator SBR, concluindo que ambos os corantes se comportaram estatisticamente da mesma forma na remoção de carbono orgânico total (COT), com valor médio de remoção de 92,02\% para C1 e 94,60\% para C2 com carregamento de 3,0 g DQO/L.d e 96,69\% para C1 e 98,30\% para C2 com carregamento de 7,0 g DQO/L.d. A biomassa apresentou baixo SVI, indicando boa sedimentabilidade para ambos os testes realizados com C1 e C2. Por fim, os microrganismos identificados na biomassa permitiram corroborar a eficiência do sistema de tratamento, uma vez que abundaram rotíferos e ciliados fixos com C1 e ciliados livres com C2, que são indicadores de boa eficiência nos processos de tratamento e, por sua vez, elevada idade do lodo. indicadores, o que contrasta com a idade do lodo utilizado neste estudo $(\theta c=15 \mathrm{~d})$.
\end{abstract}

Palavras-Chave: Corantes; Matéria orgânica; Processos biológicos; Reator aeróbico; Reator anaeróbico. 


\section{Introducción}

Los seres humanos han desarrollado a lo largo de los años diferentes procesos industriales que van de la mano con el consumo de agua, estas actividades hoy en día representan uno de los problemas ambientales de mayor importancia en el mundo, debido a la notable reducción del recurso hídrico y a los problemas en la salud pública que acarrea su descontrolado uso, poco aprovechamiento y mala disposición final [1]. En la actualidad se han producido más de 10,000 clases de colorantes, utilizados principalmente en la industria del teñido de prendas, objetos y flores [2], con la problemática de que muchas de las moléculas presentes en estos colorantes son resistentes a la acción de la luz, temperatura y a la degradación microbiana, convirtiéndolos en contaminantes altamente persistentes y perjudiciales para el ambiente [3]. Ahora bien, una gran proporción de los colorantes empleados en el teñido de tejidos, no son directamente tóxicos para los organismos vivos; sin embargo, la fuerte coloración que imparten a los medios de descarga pueden llegar a suprimir los procesos fotosintéticos en los cuerpos de agua e implica un alto riesgo a la salud humana y al ecosistema debido a que algunos de estos colorantes o sus subproductos son altamente tóxicos, mutagénicos o carcinogénicos, haciendo necesario que su presencia sea controlada [4]. La industria textil es una de las principales consumidoras de colorantes sintéticos a nivel mundial [5] y es considerada una de las industrias más contaminantes, ya que genera grandes cantidades de residuos líquidos y a su vez, emplea elevados volúmenes de agua, energía y reactivos químicos para su funcionamiento diario [7]. Por otro lado, existen otras actividades económicas asociadas al uso de colorantes sintéticos, como son los productores de cultivos de flores, los cuales emplean colorantes para teñir las flores, lo que las hace más atractivas para el consumidor [6]. En la industria de la floricultura se embellecen a las flores empleando la tinción por absorción, donde los colorantes deben ser valorados respecto a la afinidad que ellos tengan con los tejidos de la flor y deben ser altamente solubles en agua, de esta manera es importante evaluar las características principales de $\mathrm{pH}$, solubilidad e higroscopicidad previo a su uso [8].

Para lograr el teñido de las flores, éstas son introducidas en una solución acuosa que contiene el colorante, lo que da lugar a un residuo líquido, el cual no puede ser vertido al recurso hídrico sin un previo tratamiento. Por ello, para mitigar la problemática ambiental, es conveniente acudir a sistemas de tratamientos que logren reducir el grado de contaminación de forma eficiente, económica y en lo posible mediante el uso de procesos biológicos, ya que estos permiten tratar el agua sin la adición de agentes químicos, convirtiéndolos en muchos casos en tecnologías sostenibles para el tratamiento de las aguas [9]. Los procesos biológicos de depuración de las aguas residuales utilizan reacciones asociadas con los microorganismos, donde la materia orgánica y los compuestos de nitrógeno y fosforo son eliminados del agua residual por acción de las bacterias presentes, las cuales emplean la materia orgánica con donante de electrones, tanto para producir material celular como para obtener la energía necesaria para su respiración [10], y de esta manera, transforman la materia orgánica a formas inorgánicas las cuales son más inocuas para el medio ambiente [11].

La experiencia ha mostrado que la implementación de los sistemas SBR (Sequencing Batch Reactorpor sus siglas en inglés y Reactor Secuencial en Discontinuo - en español) para el tratamiento de las aguas residuales contaminadas con colorantes, ha dejado resultados confiables en la remoción de estos, facilitando su manipulación, debido a que los SBR son sistemas automatizados, en los cuales se logra una gran flexibilidad ante fluctuaciones de caudal y concentraciones del afluente [12, 13]. Los sistemas SBR son conocidos como sistemas de tratamiento de lodos activados en discontinuo, en el cual el agua residual es adicionada en un solo reactor que trabaja por cargas repitiendo un ciclo o secuencia a lo largo del tiempo, cada una con un periodo de tiempo definido [14, 15]. Las fases secuenciales convencionales de un reactor SBR son: llenado; etapa en la que se suelen combinar fases aeróbicas (en presencia de oxigeno), anóxicas (con agitación) o estáticas (sin aireación y sin agitación), según las características de las aguas residuales a tratar. La duración del llenado suele ser un $25 \%$ del total del ciclo y en esta fase se producen procesos importantes para la eliminación biológica de nutrientes y también se elimina parte de la materia orgánica biodegradable que entra en el afluente [16, 17], reacción; esta es una etapa aireada y con buena mezcla, en la que se produce la eliminación biológica de la materia orgánica biodegradable. La duración de 
este periodo suele ser de un $35 \%$ del ciclo [18], sedimentación; en esta etapa se separa el lodo del agua tratada [19], vaciado; se descarga el efluente hasta dejar el volumen inicial en el reactor [20] e inactividad; normalmente se aprovecha este tiempo de espera para realizar la purga y así mantener la edad de lodos del reactor. Las fases se combinan de diferentes formas dependiendo del objetivo de calidad que se requiere en el agua efluente, de esta manera, este proceso es empleado para el tratamiento de aguas residuales debido a sus óptimos resultados registrados en la práctica [17].

En este estudio se evaluó la eficiencia de un reactor SBR sobre la remoción de la materia orgánica, empleando un agua residual sintética contaminada con dos tipos de colorantes específicos provenientes de la industria del teñido de flores, a partir de análisis fisicoquímicos y de la biomasa presente en el reactor.

\section{Materiales y métodos}

\section{Reactor SBR}

Los experimentos fueron llevados a cabo en un reactor SBR (Figura 1), con un volumen útil de $12 \mathrm{~L}$ y una relación altura/diámetro de 4,7 . El reactor tenía una (1) válvula de entrada del afluente, dos (2) válvulas situadas a lo largo del reactor para permitir la toma de muestras, una (1) válvula de purga en el fondo del reactor y una (1) válvula de salida del efluente. La aireación era generada mediante tres (3) bombas de aire cada una con una capacidad de oxigenación $1200 \mathrm{cc} /$ min y dos (2) difusores de burbuja fina, para un total de seis (6) difusores, los cuales mantenían la concentración de oxigeno disuelto en el reactor entre 2-4 mg/L. La agitación era realizada con un agitador de varilla (Heidolph) a 40 RPM. El afluente ingresaba al reactor mediante una bomba peristáltica (Masterflex) con una caudal de $50 \mathrm{ml} /$ min y el efluente era descargado mediante una electroválvula que permitía la salida automática de este. La edad de los lodos empleada fue $15 \mathrm{~d}$.

Todas las partes descritas se encontraban conectadas a un controlador lógico programable (PLC), mediante el cual se programaron tanto las etapas como los tiempos para el funcionamiento del reactor, de esta manera, la fase llenado se realizó de forma estática durante $2 \mathrm{~h}$, la etapa de reacción se realizó durante 8 h $(4$ h de aireación y $4 \mathrm{~h}$ de mezcla) y finalmente la sedimentación se llevó a cabo durante $2 \mathrm{~h}$, para un total de 12 h por ciclo y 2 ciclos al día. Estas condiciones de operación promueven no solo la remoción material carbonáceo, sino también de material nitrogenaceo, debido a que favorece la formación de etapas combinadas aerobias y anóxicas dentro del mismo sistema.

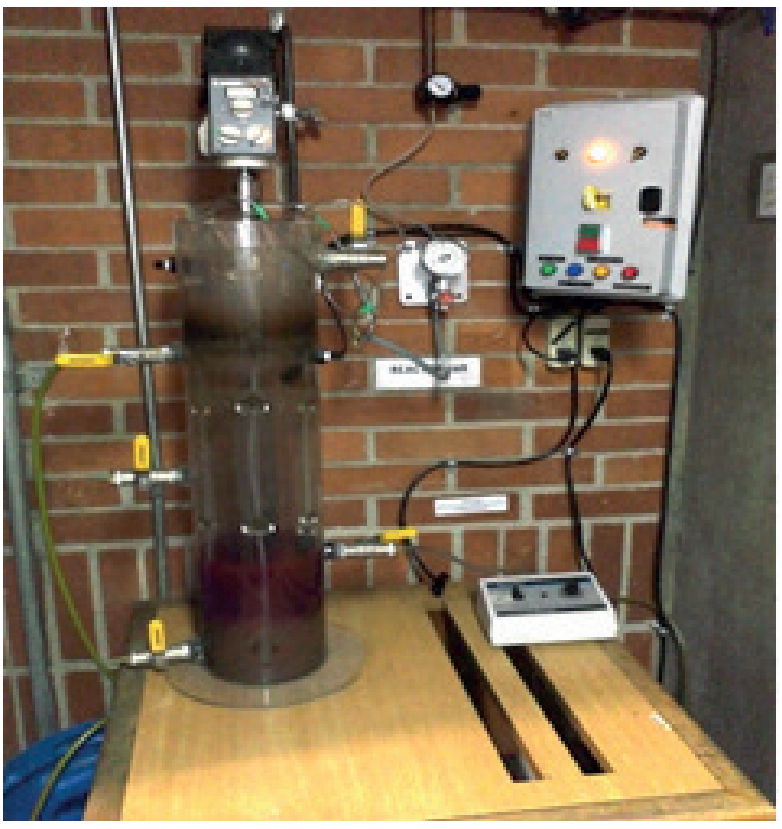

Figura 1. Reactor SBR a escala piloto para el tratamiento de colorantes

\section{Caracterización de la biomasa}

El reactor fue inoculado con lodo floculento proveniente de un reactor SBR empleado en el tratamiento de las aguas residuales domésticas en el municipio de San Jerónimo, Antioquia. La caracterización inicial del lodo arrojó una concentración de sólidos suspendidos totales (SST) de $3240 \mathrm{mg} / \mathrm{L}$ y $2576 \mathrm{mg} / \mathrm{L}$ de sólidos suspendidos volátiles (SSV), donde los SSV fueron aproximadamente el $79 \%$ de los SST, indicando un alto porcentaje de material orgánico presente. El índice volumétrico de los lodos (IVL) fue $21,45 \mathrm{ml} / \mathrm{g}$, indicando un lodo con buena sedimentabilidad (IVL $<100 \mathrm{ml} / \mathrm{g}$ ).

\section{Preparación del agua sintética}

Se prepararon $20 \mathrm{~L}$ diarios de agua sintética, compuesta por el colorante azo (C1 o C2 según el diseño de experimento), $10 \mathrm{ml}$ de macronutrientes y $10 \mathrm{ml}$ de micronutrientes. La preparación de los macronutrientes fue: $6 \mathrm{~g} / \mathrm{L} \mathrm{NH} \mathrm{N}_{4} \mathrm{Cl} ; 8 \mathrm{~g} / \mathrm{L} \mathrm{KH}_{2} \mathrm{PO}_{4} ; 16$ $\mathrm{g} / \mathrm{L} \mathrm{K}_{2} \mathrm{HPO}_{4}$. La preparación de los micronutrientes fue: $0,75 \mathrm{~g} / \mathrm{L} \mathrm{MgSO}{ }_{4} \cdot 7 \mathrm{H}_{2} \mathrm{O} ; 0,05 \mathrm{~g} / \mathrm{L} \mathrm{FeSO}{ }_{4} \cdot 7 \mathrm{H}_{2} \mathrm{O}$; 
$0,05 \mathrm{~g} / \mathrm{L} \mathrm{ZnSO}{ }_{4} ; 0,1 \mathrm{~g} / \mathrm{L} \mathrm{CaCl} \cdot 2 \mathrm{H}_{2} \mathrm{O}$ [15]. Los dos colorantes azo empleados, fueron suministrados por la empresa Cultivos de San Nicolás, dedicada al teñido de flores, ubicada en el municipio de Rionegro, Antioquia. El colorante azo C1 tenía una DQO de $1200 \mathrm{mg} / \mathrm{L}$, un $\mathrm{pH}$ de 6,58 , el $\mathrm{NH}_{4}^{+}$ de $2,30 \mathrm{mg} / \mathrm{L}$, el fosforo total menor al límite de detección $(<0,1 \mathrm{mg} \mathrm{P} / \mathrm{L})$ y el color era naranjado. El colorante azo C2 tenía una DQO de $1220 \mathrm{mg} / \mathrm{L}$, un $\mathrm{pH}$ de 6.8 , el $\mathrm{NH}_{4}^{+}$de $3,14 \mathrm{mg} / \mathrm{L}$, el fosforo total menor al límite de detección $(<0,1 \mathrm{mg} P / L)$ y el color era púrpura.

\section{Diseño de experimentos con los colorantes}

El arranque y estabilización del reactor se realizó durante 2 semanas, empleando como sustrato la dextrosa, con el fin de permitir la adaptación de los microorganismos a sus nuevas condiciones de funcionamiento. Posteriormente, los ensayos consistieron en el análisis de dos (2) cargas diferentes para cada colorante ( $\mathrm{C} 1$ y $\mathrm{C} 2)$. El diseño de experimentos que se llevó a cabo ocurrió en dos fases; la fase 1 en la que se adicionó el colorante C1 con una carga orgánica volumétrica de $3,0 \mathrm{~g}$ DQO/L.d y 7,0 g DQO/L.d (Cada carga se evaluó durante aproximadamente 30 días). Posterior a esto, se realizó un tiempo de espera de una semana donde se alimentaba solo con dextrosa para evitar dejar trazas de $\mathrm{C} 1$ en el reactor. En la fase 2 se adicionó el colorante C2 empleando las mismas cargas previamente evaluadas para $\mathrm{C} 1$.

\section{Análisis fisicoquímicos}

Para el análisis de los parámetros in situ se utilizó un equipo multiparamétrico HQ40d para el seguimiento del oxígeno disuelto dentro del reactor y un pHmetro WTW pH 7110 para la lectura del $\mathrm{pH}$. Adicionalmente, se analizaron los parámetros fisicoquímicos de Demanda Química de Oxígeno (DQO), Nitrógeno Amoniacal $\left(\mathrm{NH}_{4}^{+}\right)$y Fósforo total en el afluente y efluente del reactor y Sólidos Suspendidos Totales (SST), Sólidos Suspendidos Volátiles (SSV) en la biomasa, siguiendo los criterios analíticos del Estándar Métodos [21]. Para la determinación del Carbono Orgánico Disuelto (COT) se empleó el método de combustión a alta temperatura, filtrando previamente la muestra a analizar a través de un filtro de membrana de Nylon de $0,45 \mu \mathrm{m}$ en viales de $40 \mathrm{~mL}$ previamente muflados a una temperatura aproximada de $550^{\circ} \mathrm{C}$ durante al menos una hora, luego cada uno de los viales fueron preservados a $\mathrm{pH}<2$ utilizando ácido fosfórico $\mathrm{H}_{3} \mathrm{PO}_{4}$, para ser llevados a su posterior lectura en el analizador de carbono orgánico total Apollo 9000.

En el caso de la biomasa contenida en el reactor, se hicieron también observaciones del lodo aerobio empleando un microscopio Axioplan Universal Zeiss para epifluorescencia y luz transmitida con el fin de determinar el estado de los lodos, además, se calculó el Índice Volumétrico de Lodos (IVL) para evaluar la sedimentabilidad de la biomasa que se formaba en el reactor (Rodriguez y Peñuela, 2017). Todos los análisis fueron realizados en el laboratorio del grupo Diagnostico y Control de la Contaminación (GDCON), laboratorio acreditado para el análisis de aguas por el IDEAM bajo la norma NTC-ISO/IEC 17025, Resolución 1665 del 12 de julio de 2011.

\section{Análisis Estadísticos}

Para observar la variación en el tiempo de los diferentes puntos de muestreo en las plantas de tratamiento, se hizo un análisis exploratorio de los datos, gráficas de cajas y bigotes y correlaciones entre las variables estudiadas. Los resultados se representan como el promedio \pm desviación estándar. El análisis estadístico fue realizado empleando el software Statgraphics plus 5.1.

\section{Resultados y discusión}

\section{Análisis de eficiencias}

La Figura 2 muestra los resultados de las eficiencias obtenidas con los colorantes C1 y C2, para cada una de las cargas aplicadas. Con la carga 1 (3,0 g DQO/L.d), las eficiencias fueron menores con ambos colorantes comparado con las eficiencias obtenidas empleando la carga $2(7,0 \mathrm{~g}$ DQO/L.d). Los valores promedio fueron 92,02\% (C1) y $94,60 \%$ (C2) con la carga 1 y $96,69 \%$ (C1) y $98,30 \%$ (C2) con la carga 2 . Estas altas eficiencias encontradas indican que los reactores SBR son sistemas adecuados para el tratamiento de este tipo de aguas, ya que todas las eficiencias fueron superiores al $90 \%$ independientemente de la carga aplicada y, por otro lado, comparando las cargas, se evidenció que con la carga 2 es posible potenciar las reacciones metabólicas realizadas por los microorganismos y que dicha carga, más que inhibir los procesos, funciona como fuente de energía para llevar a cabo los procesos metabólicos de catabolismo y anabolismo; en este sentido, Cobos y González [22] definieron que algunos microorganismos son capaces de crecer usando los colorantes azo como única fuente de 
carbono, donde, estos realizan el rompimiento del enlace azo y utilizan las aminas formadas como fuente de carbono y energía para su crecimiento, adicionalmente, Coughlin et al. [23] sugirieron en su estudio que, los microorganismos adaptados a este tipo de sustrato (Colorantes azo), pueden sintetizar azoreductasas, las cuales se encargan de romper los enlaces azo en presencia de oxígeno molecular, siendo esta una de las vías de degradación de estos colorantes.

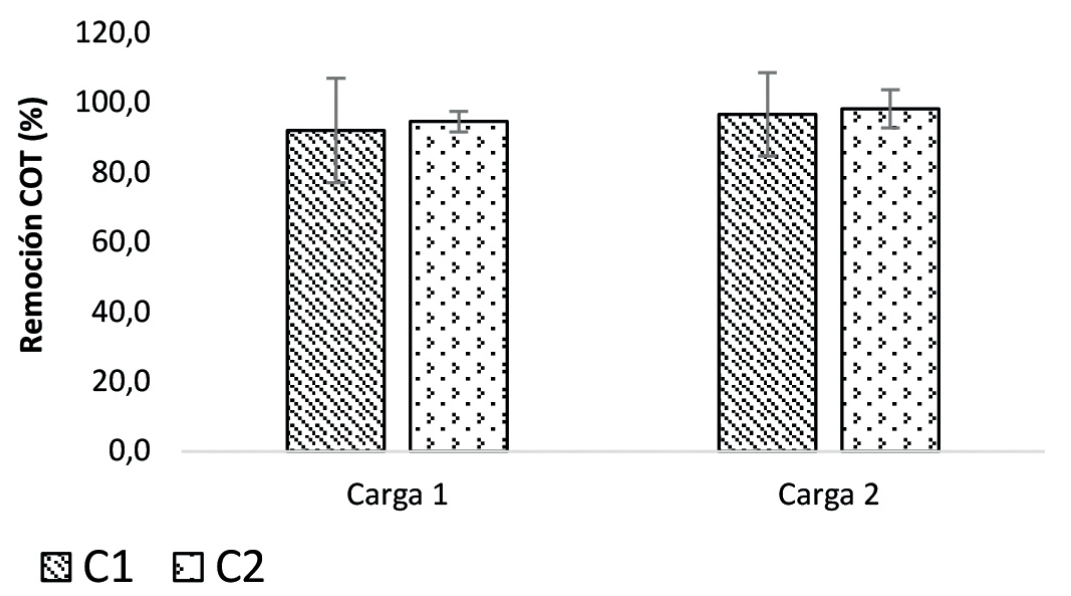

Figura 2. Eficiencia de remoción del carbono orgánico total (COT).

Comparando el comportamiento de los colorantes C1 y C2 (Figura 3), se encuentra que las mayores remociones de COT ocurrieron con el colorante C2, con un promedio de $96,45 \%$, frente a $94,31 \%$ para $\mathrm{C} 1$, estos resultados permiten establecer que la composición del colorante C1 podría contener agentes químicos inhibitorios de los procesos biológicos, lo que podría explicar, la ligera diferencia de remoción entre ambos colorantes. Por otro lado, para C1, se encontraron algunos valores atípicos $(85,21 \%$ y $86,43 \%)$ y una desviación de los datos mucho mayor, comparado con la desviación para C2, indicando que el reactor operado con $\mathrm{C} 1$ pudo sufrir importantes variaciones, lo que sustenta la hipótesis de un compuesto inhibitorio en su composición. Se tiene también, que para C1, la mayoría de los valores estaban por debajo de la media, contrario a C2, donde la mayoría de los valores estaban por encima de ésta. Estadísticamente, la mediana para ambos colorantes tuvo un comportamiento igual, independiente de las condiciones de operación del reactor y el tipo de colorante usado.

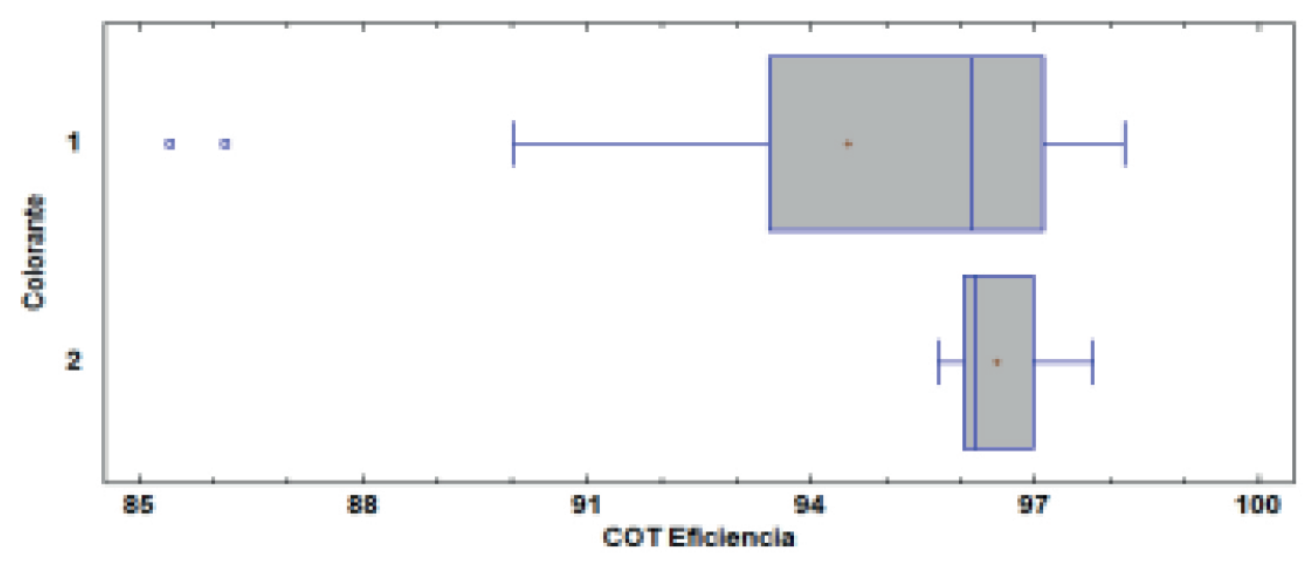

Figura 3. Comparación de eficiencias de remoción para los colorantes C1 y C2. 
La Tabla 1 presenta el análisis estadístico para los porcentajes de remoción representados en términos de COT, para los dos colorantes evaluados con sus respectivas cargas orgánicas. La varianza $\left(\sigma^{2}\right)$ de C2 fue 0,545 , indicando un comportamiento más eficiente de remoción del COT, en comparación al colorante $\mathrm{C} 1$, donde la varianza fue 15,674. Esta afirmación se corrobora con la desviación estándar, obteniéndose nuevamente un menor valor para C2 $(\sigma=0,738)$, comparado con C1 ( $\sigma=3,959)$, lo que indica que tiene un comportamiento más homogéneo respecto a la mediana que el que describe el colorante $\mathrm{C} 1$. Los valores que se muestran del rango para los dos tipos de colorantes permiten analizar la dispersión de sus datos, donde la mayor dispersión se ocurrió para el colorante $\mathrm{C} 1$, con un rango de 12,770, en comparación con el colorante C2 donde el rango fue mucho menor $(2,082)$.

Tabla 1. Resumen estadístico de la eficiencia COT por colorante.

\begin{tabular}{cccccccc}
\hline Colorante & Promedio & Mediana & Varianza & $\begin{array}{c}\text { Desviación } \\
\text { Estándar }\end{array}$ & Mínimo & Máximo & Rango \\
\hline C1 & 94,31 & 96,161 & 15,674 & 3,959 & 85,420 & 98,190 & 12,770 \\
C2 & 96,45 & 96,208 & 0,545 & 0,738 & 95,695 & 97,777 & 2,082 \\
Total & 95,156 & 96,172 & 11,319 & 3,364 & 85,420 & 98,190 & 12,770 \\
\hline
\end{tabular}

En la Figura 4 se hace un análisis del comportamiento general del sistema SBR, en ésta se observa que hay un sesgo significativo desplazado hacia la izquierda, queriendo decir con esto, que gran parte de los datos están por debajo del valor de la mediana de $96,172 \%$, por lo tanto, los valores obtenidos para el experimento en general, tales como: la varianza, la desviación estándar y el rango, permiten clasificar la eficiencia del reactor en términos de remoción del COT como un tratamiento eficiente en cuanto a remoción de materia orgánica. Dado que el $p$-valor es menor a 0,05 , la hipótesis nula (comportamiento igual para ambos colorantes) se acepta, teniéndose entonces, que el reactor SBR es capaz de remover la materia orgánica generada a partir del agua residual sintética tanto para el colorante $\mathrm{C} 1$ como para el colorante C2. Autores como Sathian et al. [24], encontraron eficiencias del orden de $79,4 \%$ para la remoción de materia orgánica en un reactor SBR con ciclos de 24 horas compuestos por llenado, reacción, sedimentación y espera, empleando aguas residuales de una industria de colorantes, por otro lado, Ong et al. [25] evaluaron la eficiencia en un reactor SBR para la remoción materia orgánica en aguas residuales con el colorante Naranja II, con eficiencias promedio del $80 \%$. Finalmente, Khosravi et al. [26], obtuvieron eficiencias del orden de $98 \%$ en un reactor SBR para la remoción de materia orgánica en aguas residuales de una empresa textil con el colorante Ftalocianina, lo anterior demuestra la alta eficacia que tienen los reactores SBR en la remoción de materia orgánica en presencia de colorantes.

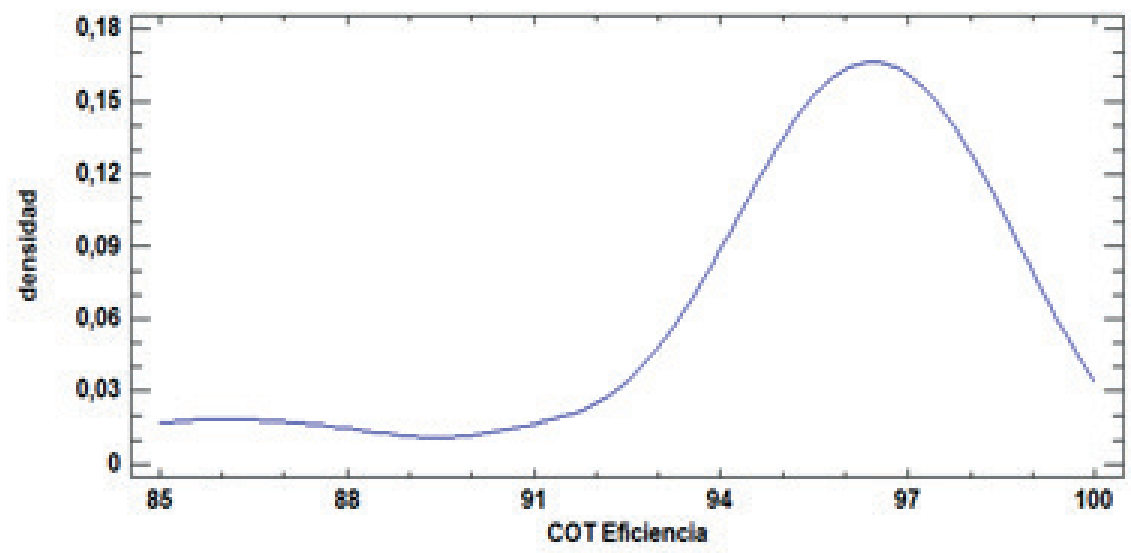

Figura 4. Distribución general del COT para el reactor SBR. 
En promedio la relación C:N:P para C1 fue $100: 2.73: 0.64$ y $100: 3.96: 0.78$ para las cargas 1 y 2 , respectivamente, y para C2 de 100:4.13:0.74 para la carga 1 y 100:5.10:0.84 para la carga 2 . En los reactores aerobios la relación óptima se ha establecido en valores de 100:5:1 [27], siendo este el balance ideal, con el cual los microorganismos adquieren los macronutrientes necesarios para incorporar a la célula y realizan el proceso de degradación de la materia orgánica de forma eficiente. En este sentido, al emplear el colorante $\mathrm{C} 1$, el balance tanto con la carga 1 como 2 fue menor al teórico, mientras que con $\mathrm{C} 2$ el balance fue más elevado con valores más cercanos a la teoría, lo que sustenta las mayores eficiencias alcanzadas con ese colorante. Es importante destacar que los colorantes azo se caracterizan por tener en su estructura el grupo azo $-\mathrm{N}=\mathrm{N}$ - como cromóforo asociado a grupos auxocromos $\left(-\mathrm{NH}_{3}\right.$, $-\mathrm{COOH},-\mathrm{OH},-\mathrm{NHR},-\mathrm{SO}_{3} \mathrm{Na}$ ) [22], por esta razón, es importante la determinación de los nutrientes y en especial del nitrógeno, el cual hace parte de la estructura de estos colorantes.

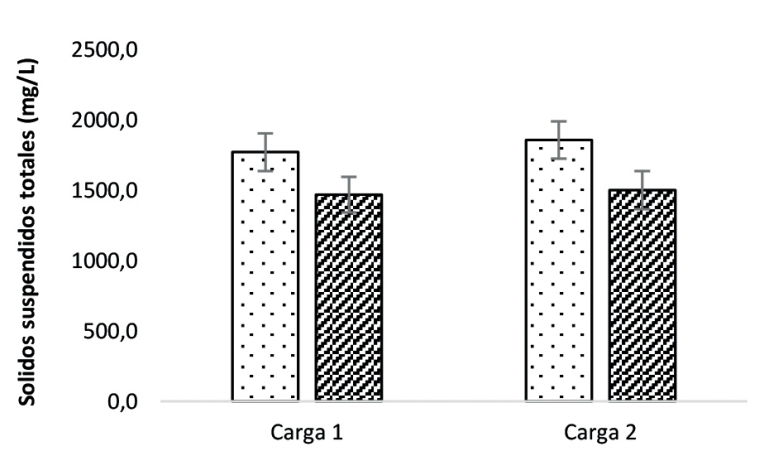

घSST C1 aSSV C1

\section{Análisis de la biomasa}

Se encontraron concentraciones promedio de SST con el colorante C1, del orden de $1770 \mathrm{mg} / \mathrm{L}$ y 1856 $\mathrm{mg} / \mathrm{L}$ para las cargas 1 y 2 , respectivamente, y de SSV de $1467 \mathrm{mg} / \mathrm{L}$ para la carga 1 y $1501 \mathrm{mg} / \mathrm{L}$ para la carga 2 (Figura 5a). En cuanto al colorante $\mathrm{C} 2$, las concentraciones promedio de SST fueron $1898 \mathrm{mg} / \mathrm{L}$ y $1932 \mathrm{mg} / \mathrm{L}$ para las cargas 1 y 2, respectivamente, y $1605 \mathrm{mg} / \mathrm{L}$ y $1732 \mathrm{mg} / \mathrm{L}$ para los SSV con las cargas 1 y 2 , respectivamente (Figura 5b). Estos resultados demuestran que la población de microorganismos fue menor cuando se empleó el colorante $\mathrm{C} 1$ en el reactor SBR y esta aumentó, cuando se empleó el colorante $\mathrm{C} 2$, resultados acordes con lo encontrado en los porcentajes de remoción del COT y que evidencian que el colorante $\mathrm{C} 1$ constituye un limitante en el crecimiento de los microorganismos, mientras que el colorante $\mathrm{C} 2$ es incorporado en la célula fácilmente por los microorganismos, lo que permite acelerar los procesos metabólicos de catabolismo y anabolismo para el crecimiento celular.

Figura 5. Comparación de la concentración de SST y SSV. a) Colorante C1. b) Colorante C2.

La Figura 6 muestra la variación de la relación SSVISST de la biomasa analizada durante el tiempo de experimentación. Ambos colorantes tuvieron comportamientos similares en cuanto a esta relación, donde el colorante C1 tuvo una mayor cantidad de sus datos por debajo de la mediana, representada con un valor de SSVISST de 0,82 , mientras que el colorante $\mathrm{C} 2$ presentó una concentración bastante homogénea, con la mayor parte de sus datos cercanos a la mediana con un valor de SSVISST de 0,87. También se encontró que ambos colorantes tuvieron valores atípicos, tanto por debajo como por encima de la media, lo cual demuestra la variabilidad que pueden tener los microorganismos en los diferentes días de monitoreo del reactor. Comparando ambos colorantes se concluye que tanto con C1 como C2, la relación SSVISST fue en promedio la adecuada para un tratamiento biológico, ya que los resultados indicaron en la mayoría de los casos el $80 \%$ de los sólidos suspendidos presentes en el reactor eran de naturaleza orgánica, lo que hace referencia a una adecuada población de microrganismos para llevar a cabo los procesos biológicos, con mejores resultados para el colorante $\mathrm{C} 2$, lo que era de esperarse debido a las mayores eficiencias de remoción de materia orgánica presentadas. 


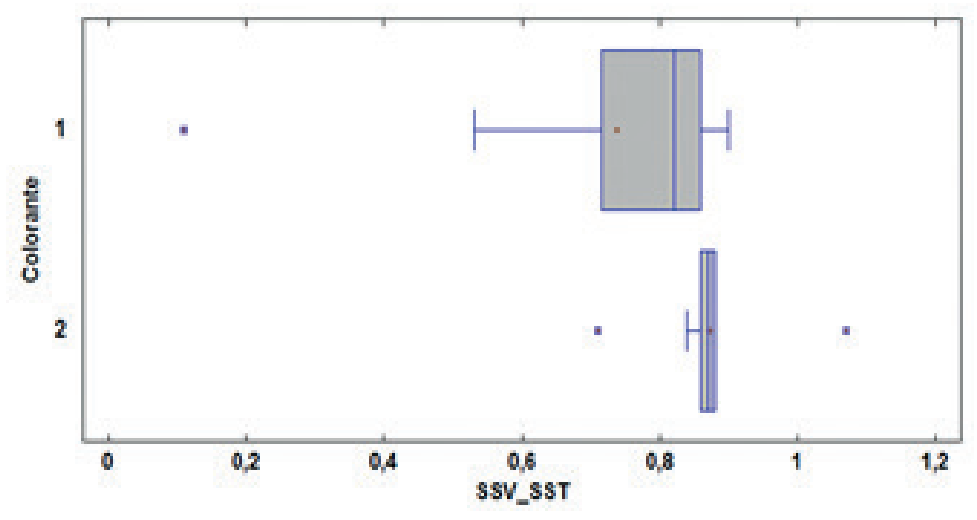

Figura 6. Relación de los SSVISST para los colorantes C1 y C2.

La Tabla 2 presenta el análisis estadístico de la biomasa en términos de relación SSVISST. La varianza $\left(\sigma^{2}\right)$ de $C 2$ fue 0,007 , valor muy por debajo de la varianza de $C 1\left(\sigma^{2}=0.050\right)$, indicando un mejor comportamiento de los microorganismos con C2 en comparación al colorante C1. Esta afirmación también se puede afirmar con la desviación estándar $(\sigma)$, donde para C2 se obtuvo un valor de 0,082 , mientras que para $\mathrm{C} 1$ fue 0,224 , indicando un comportamiento más homogéneo para $\mathrm{C} 2$, en cuanto al crecimiento de los microorganismos.

Tabla 2. Resumen estadístico para SSVISST por colorante.

\begin{tabular}{cccccccc}
\hline Colorante & Promedio & Mediana & Varianza & $\begin{array}{c}\text { Desviación } \\
\text { Estándar }\end{array}$ & Mínimo & Máximo & Rango \\
\hline C1 & 0,738 & 0,820 & 0,050 & 0,224 & 0,110 & 0,900 & 0,790 \\
C2 & 0,871 & 0,870 & 0,007 & 0,082 & 0,710 & 1,070 & 0,360 \\
Total & 0,802 & 0,860 & 0,033 & 0,181 & 0,110 & 1,070 & 0,960 \\
\hline
\end{tabular}

La tendencia general de la relación SSV/ SST (Figura 7), indica que parte de los datos analizados se ubicaron por debajo del valor de la mediana, aunque es un sesgo poco significativo. El promedio general de la relación SSVISST fue 0,802 , indicando con esto que aproximadamente el $80 \%$ de los sólidos presentes en el reactor SBR son de naturaleza orgánica, o sea que es un lodo con una alta concentración de microorganismos, hecho que favorece la depuración del agua del afluente.

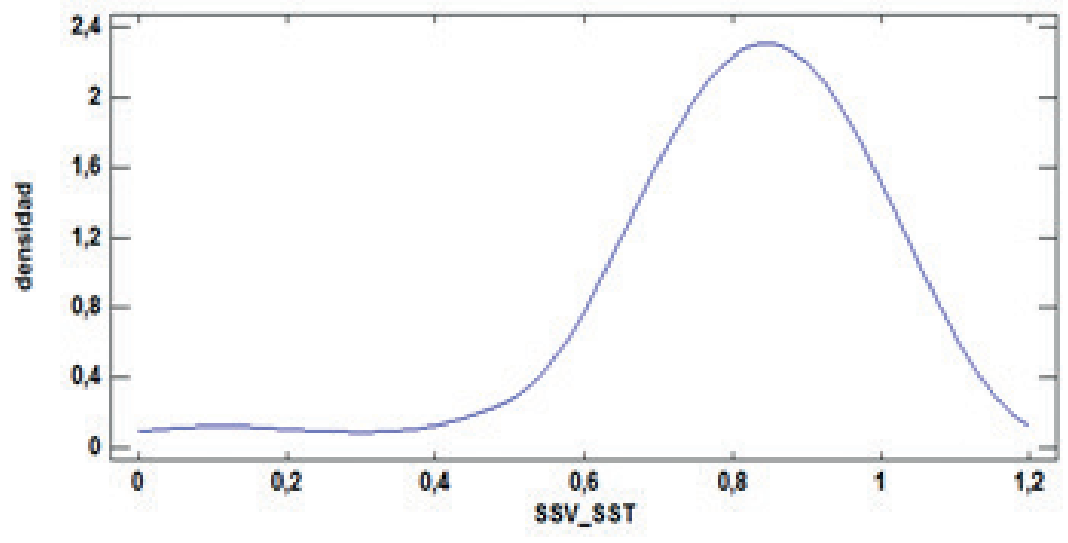

Figura 7. Distribución general de SSVISST. 
Dados los resultados obtenidos para los ensayos de sedimentabilidad empleando como indicador el IVL (Figura 8), el valor promedio para el colorante C1 fue $49,792(\mathrm{ml} / \mathrm{g})$ y para el colorante C2 fue $63,233(\mathrm{ml} / \mathrm{g})$, indicando para ambos una buena sedimentabilidad debido a que los valores fueron menores a $100 \mathrm{ml} / \mathrm{g}$ [27], estos resultados son importantes, ya que la sedimentabilidad del lodo mejora la clarificación del efluente y por lo tanto, la eficiencia final del sistema. Estudios realizados por Sathian et al. [24], encontraron valores de IVL en el rango de $90-103 \mathrm{ml} / \mathrm{g}$ para un reactor SBR empleado en la remoción de colorantes de una industria textil, lo que corrobora la buena sedimentabilidad que se logra obtener con este tipo de reactores.

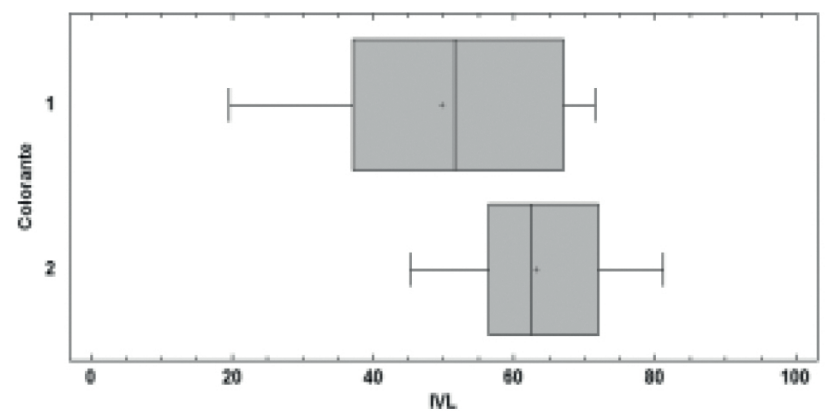

Figura 8. Representación del IVL.

En cuanto al análisis estadístico (Tabla 3), el colorante $\mathrm{C} 2$ tuvo un comportamiento más homogéneo del IVL, al igual que los parámetros previamente analizados, donde la varianza $\left(\sigma^{2}\right)$ de C2 fue 154,484, valor muy por debajo de la varianza de C1 $\left(\sigma^{2}=377,113\right)$ y la desviación estándar $(\sigma)$ fue 12,389 para C2 y 19,419 para C1, indicando que los microorganismos mantuvieron una sedimentabilidad más constante y mejor cuando operó con el colorante C2.

Tabla 3. Resumen estadístico para IVL para C1 y C2.

\begin{tabular}{ccccccccc}
\hline Colorante & Recuento & Promedio & Mediana & Varianza & $\begin{array}{c}\text { Desviación } \\
\text { Estándar }\end{array}$ & Mínimo & Máximo & Rango \\
\hline C1 & 6 & 49,702 & 51,570 & 377,113 & 19,419 & 19,530 & 71,430 & 51,900 \\
\hline C2 & 6 & 63,233 & 62,350 & 153,484 & 12,389 & 45,300 & 81,140 & 35,800 \\
\hline Total & 12 & 56,468 & 59,310 & 291,118 & 17,062 & 19,530 & 81,140 & 61,600 \\
\hline
\end{tabular}

La Figura 9 permite observar el comportamiento general del parámetro IVL en el sistema SBR, con un sesgo poco significativo desplazado hacia la izquierda, lo que indica, que algunos datos se encuentran por debajo del valor de la mediana de
59,310. En general, como se demostró mediante el análisis estadístico, el IVL tuvo un comportamiento bastante homogéneo y óptimo para tratamientos de aguas residuales con este tipo de contaminantes.

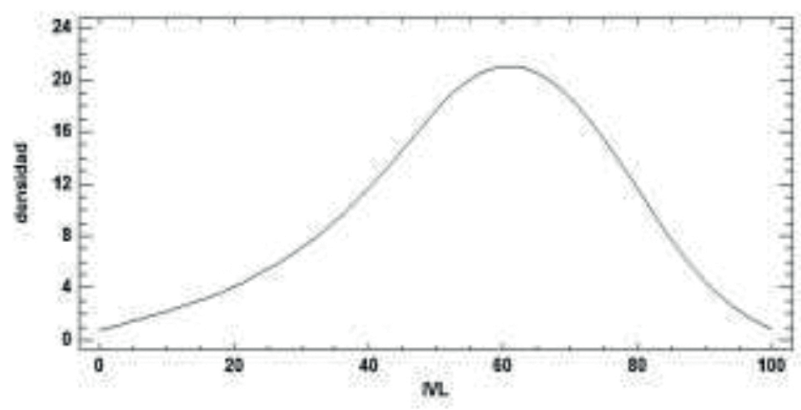

Figura 9. Distribución general del IVL. 
La presencia de ciertas especies de protozoos en los tratamientos de aguas residuales brinda información que permite identificar la efectividad de los procesos, consiguiendo de esta manera, monitorear y controlar eventuales dificultades que se presenten en el continuo proceso de funcionamiento. En el monitoreo llevado a cabo al reactor SBR, se identificaron un conjunto de organismos que comparados con la literatura permitieron afirmar que el sistema de tratamiento estaba logrando los objetivos propuestos. Los protozoos encontrados e identificados en el lodo se muestran en la Figura 10, donde la mayor abundancia para el reactor SBR operado con C1 fue de Ciliados fijos y Rotíferos (Figura 10a), mientras que para las condiciones de $\mathrm{C} 2$, abundaron principalmente los Ciliados libres (Figura 10b). En cuanto a los Rotíferos y Ciliados fijos encontrados con $\mathrm{C} 1$, estos protozoos son indicadores de buena sedimentabilidad, ya que ellos contribuyen a la remoción de las bacterias suspendidas o aquellas no floculadas, y ayudan a la formación del floc, permitiendo con esto que se logre una adecuada clarificación del efluente [28]. Sin embargo, la presencia de ellos también es un indicador de baja concentración de materia orgánica disponible lo que da lugar a procesos de respiración endógena y una edad de lodos elevada, lo que conlleva a un exceso de microorganismos viejos, los cuales tienen poca actividad, esta situación se vio reflejada en las remociones encontradas en $\mathrm{C} 1$, las cuales a pesar de ser elevadas comparadas con otros estudios, fueron menores comparadas con el colorante C2. En cuando a los Ciliados libres, estos protozoos son controladores de las bacterias filamentosas, las cuales pueden causar problemas de sedimentabilidad, esto explica los óptimos valores de IVL encontrados en C2, además, estos ciliados son indicadores de la existencia de una buena oxigenación en el reactor [28] y un buen balance de materia orgánica, lo que mejora la eficiencia de los reactores. Los Ciliados libres son considerados la condición más óptima de un proceso de remoción aerobio.

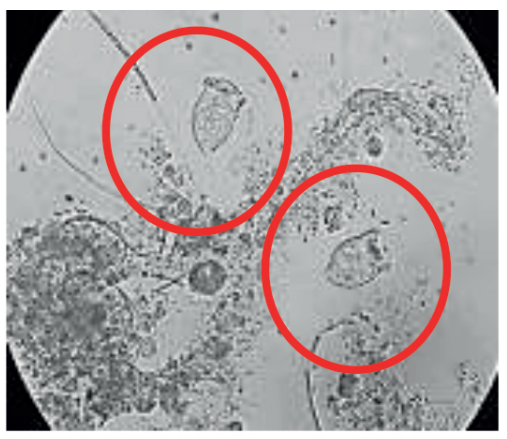

a.

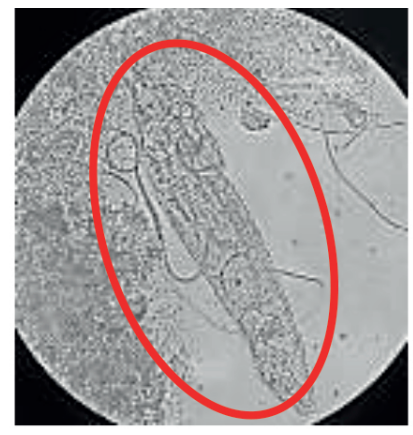

Figura 10. Protozoos encontrados con mayor abundancia en la biomasa del reactor SBR. a) Ciliado fijo y Rotífero con C1. b) Ciliado libre con C2.

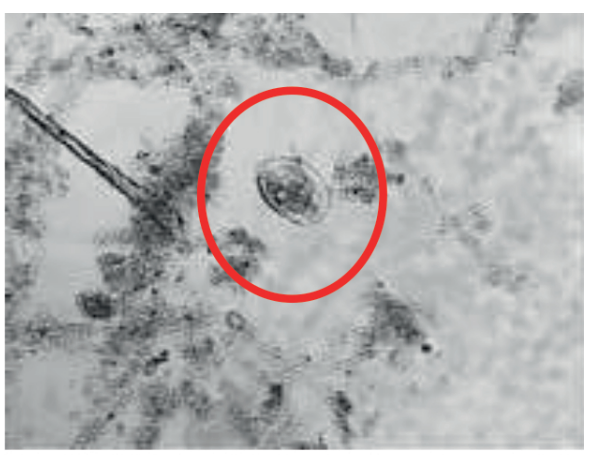

b.

\section{Conclusiones}

Los resultados de la investigación permitieron concluir que ambos colorantes se comportaron estadísticamente iguales frente a la remoción del COT, con un valor promedio de remoción de materia orgánica de $94,31 \%$ para C1 y $96,45 \%$ para C2. La relación SSVISST determinada para ambos colorantes indicó que los sólidos presentes en el reactor eran de naturaleza orgánica, hecho que favoreció la depuración del efluente. El resultado obtenido del IVL clasificó el lodo de buena sedimentabilidad, lo que contrastó con las altas eficiencias encontradas en la remoción de materia orgánica. Los microorganismos que más abundaron en la biomasa dieron certeza del buen funcionamiento del sistema SBR en cuanto a remoción de la materia orgánica. Por lo tanto, se concluye que el reactor SBR a escala piloto es altamente eficiente para remover la materia orgánica presente en aguas residuales generadas del teñido de flores, ya que es capaz de soportar la carga orgánica de los contaminantes usados en dicha actividad. Es, por esta razón, el sistema SBR una alternativa viable para el tratamiento de este tipo de aguas residuales industriales logrando un efluente de alta calidad, lo que disminuye el impacto ambiental generado por estas actividades industriales frente a los recursos hídricos. 


\section{Agradecimientos}

Los autores agradecen al grupo GDCON por la financiación de la investigación y a la empresa Cultivos de San Nicolás por la donación de los colorantes.

\section{Referencias bibliográficas}

[1] Weisburger JH. Comments on the history and important of aromatic and heterocyclic amines in public health. Mutat. Research. 2002;506507:9-20.

[2] Easton J. The dye Marke's View. Society of Dyers and colorurists Nothtingham. 1995: 9.

[3] Garcés LF, Hernández ML, Peñuela GA, Rodríguez A, Salazar JA. Degradación de aguas residuales de la industria textil por medio de fotocatálisis. Revista Lasallista de Investigación. 2006;2:15-18.

[4] Domíngues L, Pérez M, Zorrilla M, González Y, Pedrozo F. Evaluación de la remoción de colorantes mediante humedales subsuperficiales. Revista Cubana de Química. 2019;31(1):108-119.

[5] Zaruma P, Proal J, Chaires I, Salas H. Los Colorantes Textiles Industriales $Y$ Tratamientos Óptimos De Sus Efluentes De Agua Residual: Una Breve Revisión. Revista de la Facultad de Ciencias Químicas. 2019;19:38-47.

[6] Manu B, Chaudhari S. Decolorization of indigo and azo dyes in semi continuous reactors with long hydraulic retention time. Process Biochem. 2003;38:1213- 1221.

[7] Khelifi E, Ganno H, Touhami Y, Bouallagui $\mathrm{H}$, Hamdi M. Aerobic decolourization of the indigo dye containing textile wastewater using continuous combined bioreactors. J. Hazard Mater. 2008;152:683-689.

[8] Vasanth K, Ramamurthi V, Sivánesan V. Biosorption of malachite green, a cationic dye onto Pithophora sp., a freshwater alga. Dyes and Pigments. 2006;69:102-107.

[9] Tantak NP, Chaudhari. Degradation of azo dyes by sequential Fenton's oxidation and aerobic biological treatment. J. Hazard. Mater. 2006;13:698-705.

[10] García M. Puesta en marcha de un proceso de nitrificación/desnitrificación. Estudio de la influencia de la carga másica y la temperatura (tesis de maestría). Cataluña, España: Universidad Politécnica de Cataluña; 1997.

[11] Irvine RL, Wilderer PA, Flemming HC.
Controlled Unsteady State Processes and Technologies- an Overview. Water Sci. Technol. 1997;35 (1):1-10.

[12] Mahvi AH. Sequencing Batch Reactor: a promising technology in wastewater treatment, J. Environ. Health, Sci. Eng. 2008;5(2):79-90.

[13] Rodriguez DC, Pino N, Peñuela GA. Monitoring the removal of nitrogen by applying a nitrification-denitrification process in a Sequencing Batch Reactor (SBR). Bioresource Technology. 2011;102:2316-2321.

[14] Wilderer P, Irvine R, Goronsky M. Sequencing Batch Reactor Technology, Scientific and Technical Report No 10. 2001. International Water Association Publishing.

[15] Londoño Y, Peñuela GA. Biological Removal of Different Concentrations of Ibuprofen and Methylparaben in a Sequencing Batch Reactor (SBR). Water Air and Soil Pollution. 2015;226(12):393.

[16] Ferrentino R, Ferraro A, Mattei MR, Esposito G, Andreottola G. Process performance optimization and mathematical modelling of a SBR-MBBR treatment at low oxygen concentration. Process Biochem. 2018;75:230239.

[17]Barajas MG. Eliminación biológica de nutrientes en un reactor biológico secuencial (tesis de Doctorado). Barcelona, España: Escola Tècnica Superior d'Enginyers de Camins, Canals i Ports de Barcelona; 2002.

[18] Li C, Liu S, Ma T, Zheng M, Ni J. Simultaneous nitrification, denitrification and phosphorus removal in a sequencing batch reactor (SBR) under low temperature. Chemosphere. 2019;229:132-141.

[19] Ye J, Liang J, Wang L, Markou G, Jia Q. Operation optimization of a photo- sequencing batch reactor for wastewater treatment: study on influencing factors and impact on symbiotic microbial ecology. Bioresour. Technol. 2018;252:7-13.

[20] Jena J, Kumar R, Saifuddin M, Dixit A, Das T. Anoxic-aerobic SBR system for nitrate, phosphate and COD removal from highstrength wastewater and diversity study of microbial communities. Biochem. Eng. J. 2016;105:80-89.

[21] American Public Health Association (APHA), American Water Works Association (AWWA), Pollution Control Federation (WPCF). Standard methods for examination of water and wastewater 23th ed. Washington, Estados 
Unidos; 2017.

[22] Cobos YL, Gonzalez S. Degradación biologica de colorantes azo en agua residual. Conference paper. 2nd IWA Mexico Young Water Professional Conference. 2010: 1-9.

[23] Coughlin MF, Kinkle BK, Bishop JL. Degradation of azo dyes containing amino naphthol by Sphingomonas sp. strain 1CX. J. Ind. Microb. Biotechnol. 1999;23(4-5):341346.

[24] Sathian S, Rajasimman M, Radha G, Shanmugapriya V, Karthikeyan C. Performance of SBR for the treatment of textile dye wastewater: Optimization and kinetic studies. Alexandria Engineering Journal. 22014;53(2):417-426.
[25] Ong S, Toorisaka E, Hirata M, Hano T. Treatment of azo dye Orange II in aerobic and anaerobic-SBR systems. Process Biochemistry. 2005;40(8):2907-2914.

[26] Khosravi A, Karimia M, Ebrahimi H, Fallah $N$. Sequencing batch reactor/nanofiltration hybrid method for water recovery from textile wastewater contained phthalocyanine dye and anionic surfactant. Journal of Environmental Chemical Engineering. 2020;8(2):103701.

[27] Molina F, Rodríguez DC. Procesos Biológicos. Colombia: Universidad de Antioquia, Reimpresos; 2015.

[28] González G. Microbiología de los Procesos Biológicos de Tratamiento de Agua Residual. Medellín. Capítulo 8. 2012: 300-329. 